conducting a community-informed SBS adaptation using a collective decision-making process between intervention experts and local community partners will support improved safety baby shower delivery, adoption and sustainability in RUCs.

3512

Identifying Patient-Level Barriers to Non-Muscle Invasive Bladder Cancer Treatment and Surveillance Adherence in Low-Income Latino Patients: A Mixed-Methods Study

Sumeet Kaur Bhanvadia ${ }^{1}$, Siamak Daneshmand,

Mariana Stern and Lourdes Baezconde-Garbanati

${ }^{1}$ University of Southern California

OBJECTIVES/SPECIFIC AIMS: To evaluate the determinants of non-adherence to guideline treatment and surveillance and unique barriers to care in LIU Latinos with NMIBC that will inform the development of novel patient educational materials and navigation programs that could improve adherence and thus, oncologic outcomes. METHODS/STUDY POPULATION: We will recruit 40 Latino patients with new or existing NMIBC diagnoses who present to the Urology clinic at a large, tertiary public hospital in Los Angeles from November 2018 to March 2019. Quantitative (surveys) and qualitative (semi-structured interviews) data will be collected, analyzed and integrated in order to comprehensively determine patientlevel barriers to adherence. RESULTS/ANTICIPATED RESULTS: We expect to identify a unique set of patient-level barriers to adherence to NMIBC care that is unique to this population that center around 1) structural barriers to care, 2) knowledge, attitudes, and beliefs that pertain to education, acculturation, gender and values, and 3) general and disease-specific health literacy. DISCUSSION/ SIGNIFICANCE OF IMPACT: The barriers to surveillance and treatment NMIBC care are significant, particularly in LIU and minority patients, which is important as non-adherence to guideline care is linked to poorer cancer outcomes. The data generated herein will inform the development of tools and programs to aid in reducing or eliminating these barriers, but also will inform discussions on the effectiveness of current clinical practices for low-income Latino patients.

3259

Identifying the barriers and disparities for referral to kidney transplantation faced by HIV-infected patients with End Stage Renal Disease.

Ruth Adekunle ${ }^{1}$, Aneesh Mehta, MD ${ }^{1}$, Rachel Patzer, MPH, PhD ${ }^{1}$, Wendy Armstrong, $\mathrm{MD}^{1}$ and Ashley Burroughs, $\mathrm{MPH}^{1}$

${ }^{1}$ Emory University

OBJECTIVES/SPECIFIC AIMS: Our study aims to create a novel state level HIV-ESRD dataset and compare patient-level characteristics on rates of transplant referral, evaluation, waitlisting, and transplantation for HIV-positive versus HIV-negative patients. Our main hypothesis is that HIV-positive patients in Georgia are less likely to be referred to kidney transplant compared with HIVnegative patients. METHODS/STUDY POPULATION: Three datasets will be merged in order to create the HIV-ESRD dataset. The datasets are United States Renal Data System (USRDS), a southeast Transplant Referral Dataset and patient-level Georgia Department of Public Health HIV Incidence Database. The resulting study population will include patients that are older than 18 , but less than 70 , are HIV-positive and are on dialysis in Georgia. This dataset will also identify those patients who have been referred to transplantation, have been waitlisted, and have received kidney transplants between January 2012 and December 2017. If within a 1-year period, the prevalence of HIV-positive patients referred to transplant was lower than the 1-year period prevalence of HIV-negative patients for 3 consecutive years, the dialysis facility will be classified as having a within-facility disparity. We will then characterize patient level and dialysis facility-level factors that may contribute to observed findings. Patient characteristics will include demographic, clinical data, proxies of socioeconomic status, and geospatial relationships to transplant centers and rural vs urban neighborhoods. Facility-level characteristics includes profit status (profit vs. nonprofit), total number of staff (including full-time and part-time employees), aggregate demographic and clinical facility characteristics, and total number of treated patients. RESULTS/ANTICIPATED RESULTS:. We anticipate the successful creation of the proposed dataset that will allow for accurate identification of HIV-positive patients on dialysis in Georgia.. This dataset will provide the ability to determine referral, waitlisting, and transplantation rates.. We predict the overall rate of referral, waitlisting, and kidney transplantation in HIV patients will be relatively low, and that dialysis facilities with a higher proportion of HIV-positive will have lower referral rates compared to dialysis facilities treating a higher proportion of HIV-negative patients. It is foreseen that among patient-level characteristics, the strongest predictor for decreased referral rates will be HIV serostatus and among dialysis facility factors, profit status will be associated with decreased referral rates. DISCUSSION/SIGNIFICANCE OF IMPACT: This pilot study offers the creation of the first regional dataset of HIV-ESRD patients that will include patient-level characteristic of HIV-positive patients and provide a model for other states to adopt. We will contribute improved state-level description of incidence data of HIV-positive patients on dialysis, current rates of transplant referral, waitlisting, and transplantation, and offer potential associated factors that influence these processes. This knowledge will be used to determine the next steps in improving access to care; conducting qualitative research to understand dialysis facility views on transplant in HIV patients, understanding HIV patient's position on transplantation, providing education on the value of kidney transplant referral, and expanding the approach of combining patient level HIV data to the southeast.

3583

\section{Impact of Health Literacy and Risk perception on Over-the-Counter Medication Misuse}

Apoorva Reddy ${ }^{1}$ and Michelle A. Chui, PharmD, PhD

${ }^{1}$ University of Wisconsin

OBJECTIVES/SPECIFIC AIMS: This study aims to describe factors impacting older adult OTC selection and use so they can be targeted with a community pharmacy intervention to improve older adult medication safety. The primary outcome is the characterization of the relationship between health literacy, risk perception, and OTC misuse. These results will directly inform the refinement of the community pharmacy intervention such that it is tailored more precisely to the older adult patient population. METHODS/STUDY POPULATION: This cross-sectional study administers face-to-face surveys to 72 older adults (age 65+) at three locations of a massmerchandise chain pharmacy. This study is one component of a larger study to develop and implement a community pharmacy intervention 